Revista de Derecho

\title{
Ficciones disidentes reescriben la ley: investigación a través del arte sobre biopolítica, derechos de las mujeres y justicia reproductiva en Ecuador
}

Dissenting fiction re-righting law: practice-led research into biopolitics, women's rights and reproductive justice in Ecuador

\section{María Teresa Galarza Neira}

Docente Universidad Central del Ecuador

maitegalarza@yahoo.com

ORCID: https://orcid.org/0000-0003-3358-9125

DOI: https://doi.org/10.32719/26312484.2020.33.9

Fecha de recepción: 15 de marzo de 2018

Fecha de aceptación: 16 de septiembre de 2019 


\section{RESUMEN}

Este texto presenta mi investigación doctoral ${ }^{1}$ que, a través de la práctica artística de la escritura para cine, aborda cuestiones relacionadas con los derechos de las mujeres y la justicia reproductiva en Ecuador y Latinoamérica. Las preguntas básicas que articulan la reflexión son: ¿es posible crear una ficción de base legal que constituya una argumentación política sobre justicia reproductiva, evidenciando los modos en los que la ley vulnera los derechos de las mujeres en Ecuador y América Latina? $Y$, si es posible, ¿será capaz tal ficción de desafiar, cuestionar o complementar la legislación vigente, restituyendo simbólicamente los derechos vulnerados?

En esta investigación la práctica de la escritura creativa, que toma la forma de un guion cinematográfico, procura interpelar varios instrumentos internacionales de derechos humanos, la Constitución y el Código Orgánico Integral Penal del Ecuador y múltiples instrumentos de política pública ecuatoriana, con relación a los derechos de las mujeres. El guion constituye el resultado de un particular ejercicio de hermenéutica jurídica, en el que a través de la fuerza heurística que Paul Ricour reconoce a la ficción, cuatro personajes femeninos encarnan y problematizan ciertas ficciones jurídicas respecto de los derechos reproductivos en Ecuador.

La investigación propone una trayectoria del poder biopolítico para interrogar la vida como potencia y un cuerpo femenino como vida desnuda. Ficciones disidentes reescriben la ley procura hacer visibles los modos en los que la legislación ecuatoriana y sus estrategias biopolíticas de aplicación vulneran los derechos de las mujeres. En última instancia, siguiendo las ideas de Jacques Rancière, la ficción procura inducir al disenso como táctica para lograr una restitución simbólica de los derechos vulnerados.

Palabras Clave: derechos reproductivos, derechos de las mujeres, justicia reproductiva, aborto, biopolítica, investigación artística, investigación, creación.

1. La disertación titulada "Dissenting fiction re-righting law: practice-led research into biopolitics, women's rights and reproductive justice in Ecuador" es uno de los resultados de un proyecto de investigación sobre temas de justicia reproductiva conducido a través de la práctica artística. Este proyecto fue llevado a cabo por María Teresa Galarza Neira entre los años 2014 y 2017, en el Victorian College of the Arts de la Universidad de Melbourne, Australia. El guion cinematográfico The Ladies Room (en español, A puerta cerrada), escrito y reescrito por la misma autora entre el 2015 y 2017 (hasta alcanzar un quinto borrador de 106 páginas) constituyó el producto artístico que facultara la exploración creativa de la temática. Como resultado de este proyecto de investigación, que propone una suerte de hermenéutica jurídica a través de la escritura creativa, en el año 2018 María Teresa Galarza Neira obtuvo grado de PhD. El texto completo de la disertación, en su idioma original, inglés, puede consultarse en 〈https://minerva-access.unimelb.edu. au/handle/11343/210743>. Por su parte, el guion cinematográfico ha sido registrado bajo su título en inglés en la oficina de Copyrights de los Estados Unidos de América, y bajo su título en español en el Servicio Nacional de Derechos Intelectuales del Ecuador. Por la naturaleza propia de esta obra artística de carácter inédito, el acceso a su texto completo es restringido. En el año 2019 la autora inició el proceso de escritura del sexto borrador del guion. 


\begin{abstract}
This text is an introduction to my doctoral creative research ${ }^{2}$ that addresses issues of women's rights and reproductive justice in Ecuador and Latin America, through the practice of the screenplay writing. The basic research questions that allow me to articulate this reflection are: is it possible to set on legal grounds a fictional story about women's reproductive rights, in order to propose a political argumentation about women's fundamental rights and reproductive justice issues in Ecuador and Latin America? If it is, could that fiction in anyway question and contest the legislation in force in order to re-right the rights law disregarded?

Through a feature-length screenplay and accompanying dissertation, artistic practice-led research interrogates several international human rights instruments, Constitutional law, Criminal Law and public policy in Ecuador, with regards to women's reproductive rights. The screenplay is the outcome of a particular sort of juridical hermeneutics; where the heuristic force Paul Ricœur recognizes in fiction allows the screenplay writer to re-present various juridical fictions regarding reproductive rights, shaped according to the Ecuadorian law, in the form of four female characters.

The research proposes a biopolitical trajectory in order to interrogate life as possibility, potentiality, and a female body as Bare Life. Dissenting fictions re-righting law renders visible how Ecuadorian law and its biopolitical enforcement strategies disenfranchise women. It ultimately introduces dissensus, as suggested by Jacques Rancière, as a tactic to re-right those rights law disregarded.

KEYWORDS: reproductive rights, women's rights, reproductive justice, abortion, biopolitics, artistic research, practice-based research, creative research.
\end{abstract}

\title{
INTRODUCCIÓN
}

$\mathcal{H}$ icciones disidentes reescriben la ley es una introducción a mi investigación doctoral que a través de la práctica artística indaga temas de biopolítica, derechos de las mujeres y justicia reproductiva en Ecuador. Aquí, la práctica de la escritura del guion cinematográfico se convierte en un medio para articular una argumentación

2. María Teresa Galarza, "Dissenting fiction re-righting law: practice-led research into biopolitics, women's rights and reproductive justice in Ecuador" (tesis doctoral, The University of Melbourne, 2017), 〈https:// minerva-access.unimelb.edu.au/handle/11343/210743>. 
política $^{3}$ que pretende interpelar ciertos aspectos de la legislación ecuatoriana y de la política pública en materia de salud. De esta manera, el guion de largometraje titulado A puerta cerrada ${ }^{4}$ ha procurado constituirse en una táctica disidente, en el sentido que Jacques Rancière otorga a la noción de disenso. Rancière define al disenso como la "demostración (manifestación) de un vacío en lo sensible". ${ }^{5}$ En este caso, esa táctica disidente ha sido articulada alrededor de una historia que, funcionando como una "demostración política, hace visible lo que no tenía ninguna razón para ser visto". ${ }^{6}$

En Ficciones disidentes reescriben la ley, el guion cinematográfico A puerta cerra$d a$, que articula esta investigación, tiene base jurídica. Así, en este las categorías de biopolítica y justicia reproductiva, junto con elementos relevantes relacionados con los derechos de las mujeres, han sido herramientas fundamentales para la ambientación, la creación de personajes y el diseño de la trama de la historia.

Metodológicamente, las preguntas que guían mi indagación son: ¿cómo crear una ficción con base legal que articule una demostración política sobre derechos de las mujeres y justicia reproductiva en Ecuador? Como estrategia metodológica me propuse dos pasos para la creación de una ficción tal, que para efectos de esta investigación tomaría la forma de un guion cinematográfico. El primero, fue la definición de los fundamentos jurídicos para establecer la historia de A puerta cerrada. Luego de este particular ejercicio de ambientación de la historia, el segundo paso propone una constante interrelación entre narración y legislación, articulada a través de la caracterización de cuatro personajes femeninos que, respecto de la norma jurídica, operan como una suerte de hechos personificados (para Bakunin fatti personificati). ${ }^{7}$ A partir de allí, el guion procura proponer una argumentación política que, en términos de Rancière:

es al mismo tiempo la manifestación de un mundo posible en que el argumento podría contar como argumento, que es dirigida por un sujeto calificado para argumentar, sobre un objeto identificado, a un destinatario, quien es requerido para ver el objeto y oír el argumento que 'normalmente' no tiene ninguna razón para ver o escuchar. ${ }^{8}$

3. Jacques Rancière, Dissensus: On Politics and Aesthetics (London: Continuum, 2010), 38.

4. Texto inédito escrito por María Teresa Galarza Neira entre el 2015 y 2017 (hasta alcanzar un quinto borrador de 106 páginas) y titulado The Ladies Room en su versión inglesa; el guion constituye el componente creativo del proyecto doctoral de investigación basada en la práctica artística titulado "Dissenting fiction re-righting law: practice-led research into biopolitics, women's rights and reproductive justice in Ecuador".

5. Ibíd.

6. Ibíd.

7. Mijaíl Bakunin, La libertà degli uguali (Milan: Elèuthera, 2009), 86.

8. Jacques Rancière, Dissensus: On Politics and Aesthetics, 38-9. Original en inglés. Énfasis añadido. 
Un sujeto político es, para Rancière, "el operador de un dispositivo particular de subjetivación y litigio a través del cual la política comienza a existir". ${ }^{9}$ Aquí, la escritura de ficción operaría como este dispositivo de litigación que propone un acercamiento narrativo a la ley, permitiendo intervenir en ella desde las nociones de lo decible y visible. ${ }^{10}$ Este arraigo en la noción de disenso, hizo del guion un medio útil para revisar el marco legal en temas de derechos de las mujeres y justicia reproductiva, pues como señala Rancière, la ficción:

no es un término que designa lo imaginario frente a lo real; se trata del re-encuadre de lo 'real', o del encuadre de un disenso. La ficción es una forma de cambiar los modos de presentaciones sensoriales y las formas de enunciación; de diferentes encuadres, escalas y ritmos; y de la construcción de relaciones significativas entre realidad y apariencia, el individuo y el colectivo. ${ }^{11}$

\section{FUNDAMENTOS JURÍDICOS PARA CONTAR UNA HISTORIA}

El primer paso para la construcción de un guion cinematográfico que, basado en la legislación, procure interpelarla, es precisamente la definición de las normas jurídicas que servirían de fundamento de la escritura, y frente a las cuales la ficción funcionaría a modo de contestación. Ficciones disidentes reescriben la ley se concentra en la esfera de los derechos humanos, poniendo particular interés en los derechos de las mujeres, especialmente, en derechos sexuales y reproductivos, de autodeterminación y de acceso a la salud. En instrumentos internacionales de derechos humanos, en la Constitución de la República del Ecuador y en el Código Orgánico Integral Penal (COIP) se ha procurado encontrar suficientes elementos que permitan entender, representar y problematizar el posible ejercicio de esos derechos.

De los 29 numerales dedicados al reconocimiento de derechos fundamentales, incluidos en el art. 66 de la Constitución ecuatoriana, el 1 y el 10 son pertinentes para esta reflexión: "art. 66. Se reconoce y garantizará a las personas: [...] 1. El derecho a la inviolabilidad de la vida. No habrá pena de muerte. [...] 10. El derecho a tomar decisiones libres, responsables e informadas sobre su salud y vida reproductiva y a decidir cuándo y cuántas hijas e hijos tener". ${ }^{12}$

9. Ibíd.

10. Ibíd.

11. Ibíd., 141.

12. Ecuador, Constitución de la República del Ecuador, Registro Oficial 449, Suplemento, 20 de octubre de 2008. 
En el num. 1 del art. 66 se reconoce el derecho a la vida, inherente a cada ser humano, y consignado en el Pacto Internacional de Derechos Civiles y Políticos, ratificado por el Ecuador. Sin embargo, el art. 45 de la Constitución de la República del Ecuador ofrece una definición ampliada de este derecho: "Los niños y adolescentes gozarán de los derechos que son comunes a todos los seres humanos, además de los que son específicos a su edad. El Estado debe reconocer y garantizar la vida, incluyendo el cuidado y protección desde el momento de la concepción" (énfasis añadido). ${ }^{13}$ La fórmula constitucional propuesta por el art. 45 equipara la obligación estatal de garantizar el derecho a la vida antes y después del nacimiento; lo que implica proteger y cuidar esa vida, aparentemente en los mismos términos, durante el período de gestación y a lo largo de la infancia.

En este horizonte, otro elemento que debe ser tomado en consideración está incluido en el art. 44 de la Constitución ecuatoriana. Al referirse a los derechos de niños y niñas, el texto establece: "se atenderá al principio de su interés superior y sus derechos prevalecerán sobre los de las demás personas". ${ }^{14}$

Si volvemos sobre la problemática ecuación que la Constitución establece entre la vida, desde el momento de la concepción de esos niños y niñas, cuyos "derechos prevalecerán sobre los de las demás personas", es posible imaginar que un sinnúmero de derechos constitucionalmente reconocidos (especialmente los relacionados con la autodeterminación, la salud y la reproducción) dejan de ser fundamentales para las mujeres.

Esto deriva, entre otras cosas, en la tipificación del aborto consentido en el art. 149 del Código Orgánico Integral Penal del Ecuador. El COIP considera al aborto consentido como un delito contra la vida, sancionado con prisión y, en su art. 150, prevé 2 casos de aborto no punible: "1. [El] practicado para evitar un peligro para la vida o salud de la mujer embarazada y si este peligro no puede ser evitado por otros medios. 2. [Cuando] el embarazo es consecuencia de la violación en una mujer que padezca de discapacidad mental". ${ }^{15}$

13. Cabe anotar que esta garantía de protección de la vida desde la concepción, tipificada en la Constitución ecuatoriana y en varias Constituciones de Latinoamérica tiene relación directa con el reconocimiento del derecho a la vida desde la concepción, incluido en el art. cuatro, num. uno de la Convención Americana de Derechos Humanos, tal como anota Alvaro Paul, "Controversial Conceptions: The Unborn and the American Convention on Human Rights", Loyola University Chicago International Law Review, vol. 9, Issue 2 (2012): 219. "The ACHR's approach to the right to life could be considered as a guiding value of this regional system because it reflects the special importance which domestic legislation grants to the unborn in the context of the Americas. Indeed, many political constitutions-some of which were promulgated as recently as 2008 and 2010-protect life from the moment of conception".

14. Ibíd.

15. Ecuador, Código Orgánico Integral Penal, Registro Oficial 180, Suplemento, 10 de febrero de 2014, art. 150. 
Además, el código en su art. 422, num. dos, establece el deber de denunciar ${ }^{16}$ por mandato expreso de la ley, impuesto a profesionales de la salud que lleguen a conocer acerca de la comisión de un delito. Este marco jurídico ha determinado la existencia recurrente de una controversial práctica por parte de los prestadores de salud en Ecuador, la de reportar ante las autoridades a aquellas mujeres que buscan atención médica por aparentes complicaciones relacionadas con abortos consentidos y clandestinos. Como mencionan Zaragocin y otras: "La tipificación legal mencionada, junto con el contexto socio-político descrito, agudiza el clima y las prácticas de persecución a las mujeres. En varios hospitales públicos del país, el personal médico es impulsado a denunciar mujeres sospechosas de haber interrumpido sus embarazos". ${ }^{17}$

$\mathrm{Al}$ respecto cabe anotar que los esfuerzos por mapear la criminalización del aborto voluntario en Ecuador, si bien dan cuenta efectivamente de procesos de persecución en contra de las mujeres, arraigados incluso en el sistema de salud (como se verá más detalladamente en las secciones posteriores de este texto, relacionadas con cada uno de los personajes), no guardan necesariamente relación con estadísticas oficiales respecto del encarcelamiento de las mujeres judicializadas o denunciadas por haberse practicado abortos clandestinos. Esto se debe, entre otras cosas, a la labor de ONG, colectivos de mujeres y grupos de apoyo que brindan acompañamiento legal en estas causas, procurando evitar el procesamiento de las mujeres y, en caso de darse, la absolución del delito o, al menos, la sustitución de penas.

De todos modos, las múltiples consecuencias de este complejo marco jurídico que, cuando se traduce en políticas públicas operativas, sistemáticamente vulnera los derechos de mujeres, pueden ser explicadas con un ejemplo: según el reporte Indicadores básicos de salud. Ecuador 2010, publicado por el Ministerio de Salud Pública del Ecuador y basado en registros de ingresos y egresos hospitalarios del país, la quinta causa de morbilidad de la población en general y la primera causa de morbilidad de las mujeres fue "aborto no especificado". ${ }^{18}$ Dos años más tarde, en el reporte Indicadores básicos de salud. Ecuador $2012,{ }^{19}$ publicado por la misma institución, el aborto había desaparecido totalmente como causa de morbilidad. Esto sucedió en medio de una acalorada discusión nacional sobre la (des)penalización del aborto, como parte del debate legislativo acerca del nuevo Código Penal, hoy en vigencia. Probablemente en un

16. Ibíd., art. 422.

17. Sofía Zaragocin et al., "Mapeando la criminalización del aborto en el Ecuador", Revista de Bioética y Derecho, n. ${ }^{\circ} 43$ (2018): 115, «ttp://scielo.isciii.es/pdf/bioetica/n43/1886-5887-bioetica-43-00109.pdf .

18. Ministerio de Salud Pública del Ecuador, Indicadores Básicos de Salud 2010 (Quito: MSP / INEC / Organización Panamericana de la Salud, 2011), 12-4.

19. Ministerio de Salud Pública del Ecuador, Indicadores Básicos de Salud 2012 (Quito: Ministerio de Salud Pública, 2013). 
intento por evitar referencias a la incidencia y los riesgos de abortos clandestinos en Ecuador, el Ministerio de Salud Pública excluye al aborto de su reporte 2012, siendo imposible contar con datos oficiales acerca de su incidencia en términos de morbilidad y mortalidad. Si bien este documento incluye información relacionada con las muertes maternas en Ecuador, utiliza la pertenencia étnica como única variable de análisis, sin especificar las causas de tales muertes.

Así, una de las medidas del gobierno de turno frente a la incidencia de abortos en condiciones de riesgo, morbilidad y mortalidad de las mujeres en Ecuador, fue la de excluir a tal fenómeno y sus efectos de los reportes oficiales del Ministerio de Salud Pública del Ecuador. Esta política de enmascaramiento dio paso a la creación de subregistros cuya validez cuestionable contribuye a la invisibilización de las vidas y muertes de mujeres por esta causa en Ecuador. Y las autoridades ecuatorianas reconocen este ocultamiento. En el año 2017 el Plan Nacional de Salud Sexual y Salud Reproductiva, publicado por el Ministerio de Salud Pública del Ecuador, explica: "el 'aborto no especificado' representa el 68,6\% de egresos hospitalarios por esta causa. A pesar de los datos mencionados existe un alto sub registro de esta práctica". ${ }^{20}$

Este ocultamiento debe analizarse en el contexto de las discusiones respecto de la aprobación del Código Orgánico Integral Penal, particularmente a la luz de la controversia nacional que causó la amenaza del entonces primer mandatario, economista Rafael Correa Delgado, de renunciar a su cargo en caso de que la Asamblea Nacional (en la que, por entonces, su gobierno y partido tenían mayoría) diera paso a un debate legislativo acerca de la despenalización del aborto. Entre el 2010 y el 2017, la posición presidencial decididamente contraria a la despenalización del aborto consentido jugó un rol determinante respecto del rumbo de la política pública en materia de derechos sexuales y reproductivos en Ecuador.

Esto fue especialmente cierto entre el 14 de octubre de 2011, fecha de presentación del proyecto del COIP a la Asamblea Nacional por parte del ejecutivo, y el 28 de febrero de 2015, poco después de la aprobación del COIP, cuando por decreto presidencial se reemplazó la "Estrategia Nacional Intersectorial de Planificación y Prevención del Embarazo en Adolescentes (Enipla)" por el "Plan Familia Ecuador"; el primero, constituido desde una perspectiva de salud sexual y reproductiva, y el segundo, desde una perspectiva de educación en valores como estrategia de prevención del embarazo adolescente. Así, la voluntad política que gestó el COIP permeó otras esferas de la política pública, determinando este enmascaramiento de las vidas y muertes de esas

20. Ministerio de Salud Pública del Ecuador, Plan Nacional de Salud Sexual y Salud Reproductiva (Quito: Ministerio de Salud Pública, 2017), 23. 
mujeres que, en Ecuador, se someten a abortos consentidos, clandestinos y, muchas veces, inseguros.

En este escenario jurídico-político se ambienta y desarrolla la historia de "A puerta cerrada". Aquí, las normas constitucionales y legales relacionadas con ciertos temas de justicia reproductiva, y muy particularmente con la penalización del aborto consentido, son los fundamentos legales para contar esta historia de cuatro personajes femeninos: Isabel, Marcia, Alicia y Karlita, cuyas interacciones en una aislada hacienda en medio de los Andes constituyen la trama del guion.

\section{BIOPOLÍTICA Y JUSTICIA REPRODUCTIVA A CUATRO VOCES}

Los cuatro personajes femeninos de "A puerta cerrada" constituyen siluetas que se yerguen en los intersticios de la política pública ecuatoriana; en la distancia entre la tasa de fecundidad y la tasa de fecundidad deseada, entre la criminalización del aborto y sus prácticas clandestinas.

Así, Isabel, Marcia, Alicia y Karlita podrían ser una suerte de esos hechos personificados de los que habla Mijaíl Bakunin en sus reflexiones en torno a la libertad, la anarquía y la ideología. Para Bakunin:

Cada nueva generación encuentra todo listo desde el nacimiento un mundo de ideas, imágenes y sentimientos, que recibe como un legado de siglos pasados. Este mundo no se presenta al niño en su forma ideal, como un sistema de representaciones e ideas, como una religión, como una doctrina: el niño sería incapaz de darle la bienvenida y concebirlo en esta forma; el mundo de las ideas se impone a él como un mundo de hechos personificados, encarnados en las personas y en las cosas que lo rodea, como un mundo que habla a sus sentidos a través de todo lo que significa y ve. ${ }^{21}$

En cierto sentido, los personajes femeninos de "A puerta cerrada" funcionan como hechos personificados que, entre otras cosas, dan forma y voz a ciertas ficciones jurídicas; ellas encarnan ciertos supuestos legales relacionados con temas de justicia reproductiva; en su diseño, caracterización e interacciones el guion asume una perspectiva de la biopolítica. Para entender cómo operan, o lo que implican, es necesario acercarse un poco y conocer más en detalle a cada personaje.

21. Mijaíl Bakunin, La libertà degli uguali, 86. Original en italiano, la traducción es mía. 


\section{ISABEL: MATERNIDAD COERCITIVA Y UN MATERNAR IMPOSIBLE. LA VIDA COMO IMPOTENCIA}

En este (con)texto Isabel sería el cuerpo biopolítico en el que se encarna inevitablemente la norma jurídica. Isabel, en la historia, también es la practicante de abortos clandestinos, una mujer con entrenamiento en medicina, cuyo embarazo no deseado no se adecuó a las condiciones del art. 150 del COIP y fue forzada a llevarlo a término por una voluntad ajena a la suya que no pudo resistir. Dedicarse a la práctica de abortos, clandestinos, sí, pero en general seguros, es una suerte de revancha.

De todos modos, dado que los embarazos no planificados a menudo desaparecen de los informes oficiales, su incidencia, un indicador elocuente de la eficacia de políticas gubernamentales de planificación familiar, suele leerse en términos del resultado del embarazo: un parto deseado o no deseado. ${ }^{22}$ Según estadísticas oficiales sobre salud materna e infantil, en Ecuador la Tasa Global de Fecundidad (TGF) es de tres hijos o hijas por mujer de 15 a 49 años; el mismo reporte explica que la TGF Deseada es de $2,5 \mathrm{~m}$ y la TGF No Deseada es de $0,5 \cdot{ }^{23}$ Dicho de otro modo, uno de cada seis niños nacidos en Ecuador entre 2007 y $2012^{24}$ es considerado no deseado por su madre.

Dado que mediante este instrumento se establece que las mujeres ecuatorianas entre 15 y 49 años de edad tienen un promedio de tres hijos, podría decirse que una de cada dos mujeres ecuatorianas está criando un hijo o hija que no deseó tener. Y la Isabel de "A puerta cerrada" es una de ellas. A través de las interacciones de Isabel la investigación indaga: si atendiendo al marco jurídico ecuatoriano una vez confirmado el embarazo el llevarlo a término es un imperativo legal, ¿cuáles podrían ser las implicaciones de tal imposición para una mujer? ¿Puede ella ser madre de ese niño o niña cuya existencia es el testimonio de la vulneración de sus propios derechos? Si sí, ¿de qué modo?

Esta parte de la discusión se basa en el argumento de justicia reproductiva explicado por Loretta J. Ross y Rickie Solinger. Para Ross y Solinger la justicia reproductiva se fundamenta en tres principios: el derecho a tener un niño, el derecho a no tenerlo

22. Es de notar que en varios instrumentos de política pública en materia de salud pública, incluyendo los informes oficiales de la propia Encuesta Nacional de Salud y Nutrición, ENSANUT-ECU 2012, publicada por el Ministerio de Salud Pública del Ecuador, se hace pocas y vagas referencias a los embarazos, partos e hijos no deseados, y se prefiere usar la terminología de no planificados para designarlos, aunque esta no necesariamente aluda a lo mismo.

23. Ministerio de Salud Pública del Ecuador e INEC, Encuesta Nacional de Salud y Nutrición, tomo II, Salud Sexual y Reproductiva (Quito: Ministerio de Salud Pública / INEC, 2015), 119.

24. Según la metodología de la encuesta, para este indicador se tomó en cuenta únicamente a los niños nacidos vivos durante los cinco años anteriores a la ejecución de la misma. 
y el derecho de criar a los hijos e hijas que se decida tener en un ambiente sano y saludable..$^{25} \mathrm{Su}$ enfoque considera los derechos reproductivos en diálogo con la noción de justicia social, desde una perspectiva interseccional. Desde el punto de vista de este argumento de justicia reproductiva puede considerarse una forma de coerción el hecho de imponer la "maternidad en una mujer que no lo desea". ${ }^{26}$ Lo curioso es que en muchos casos esta forma de coerción ha sido legal y constitucionalmente establecida. Por ello, el argumento de justicia reproductiva defiende la necesidad social de garantizar el acceso a procedimientos de aborto seguro; al tiempo que problematiza las actuales condiciones de accesibilidad a tales procedimientos que, incluso en países del norte global, usualmente no están disponibles para mujeres de escasos recursos. ${ }^{27}$

Reflexionando en torno a las implicaciones que los nacimientos imperativos podrían tener en la vida de las mujeres, la crianza de un niño no deseado podría ser la más compleja y duradera de estas. Desde una perspectiva feminista matricéntrica, Andrea O'Reilly define lo que ella denomina los diez supuestos ideológicos de la maternidad patriarcal, uno de los cuales es la biologización que, dice O'Reilly, "posiciona a la madre biológica como 'real' y auténtica madre". ${ }^{28}$ Respecto de las madres biológicas, y en virtud de estos supuestos ideológicos, se generará la expectativa social de que proporcionen a sus hijos e hijas un cuidado socialmente aceptable, que satisfaga las tres demandas del trabajo materno identificadas por Sarah Ruddick: preservación, crecimiento, aceptabilidad social. ${ }^{29}$ Estas expectativas llevan al establecimiento de ciertos mecanismos para hacer cumplir y garantizar la calidad de la atención brindada por las madres. Como explica O'Reilly, "las madres son vigiladas por lo que Sarah Ruddick llama 'la mirada de los demás' ", ${ }^{30}$

La historia de "A puerta cerrada" muestra a Isabel tratando de gestionar su historia personal, su ocupación como practicante de abortos y la crianza de Karlita, una niña de 6 años que crece al cuidado de quienes ella cree sus tías. Isabel, involuntaria madre biológica de Karlita, es la cuidadora principal, y sus labores son vigiladas muy de cerca por Marcia.

25. Loretta Ross y Rickie Solinger, Reproductive justice. An introduction (Oakland: University of California Press, 2017), 9. Original en inglés, la traducción es mía.

26. Ibíd., 209.

27. Ibíd., 123 .

28. Andrea O'Reilly, Matricentric Feminism. Theory, Activism, and Practice (Ontario: Demeter Press, 2016), 14. Original en inglés, la traducción es mía.

29. Sara Ruddick, Maternal Thinking. Toward a Politics of Peace (Boston: Beacon Press, 1995), 17.

30. O'Reilly, Matricentric Feminism, 16. 


\section{Marcia O La VigilanCia INFinitesimal: BIOPOLÍTICA A LA HORA DE LA CENA}

Marcia representa los modos de hacer del poder biopolítico. Es la hermana y ayudante de Isabel, que no admitió un aborto en su familia y amenazó con denunciar el hecho ante las autoridades si llegaba a darse.

A través de las acciones e interacciones de Marcia en la historia, la investigación procura indagar: más allá del mandato legal y de los procedimientos establecidos por la política pública, ¿cuáles podrían ser los mecanismos para inscribir efectivamente la letra de la ley sobre el cuerpo de una mujer?

Esta pregunta nos conduce a los esfuerzos de Michel Foucault por nombrar la relación conflictiva entre poder y deseo. Como parte de esta reflexión, Foucault definió la uniformidad del aparato, la manera en la cual el poder "actúa de modo uniforme y exhaustivo en todos los niveles", a través de una serie de instituciones y dispositivos que "reproducen sin cesar los mecanismos de la ley, el tabú y la censura: del estado a la familia". ${ }^{31}$ Para avanzar, mantendremos esa idea de un ejercicio de poder que opera de manera uniforme y exhaustiva del Estado a la familia.

Aunque el guion de "A puerta cerrada" no permite a su lector entender de inmediato la relación entre Marcia e Isabel, queda claro que viven y crían juntas a Karlita. A medida que la historia se desarrolla, se revela que Isabel y Marcia son hermanas y que, por las historias previas de los personajes, Marcia representa una suerte de poder coercitivo frente a Isabel.

Isabel, como madre biológica de Karlita, asume la mayoría de obligaciones maternas respecto a ella. Mientras Marcia opera como un mecanismo de vigilancia infinitesimal, uno de esos "micro-poderes relacionados con el cuerpo" que Foucault vio erguirse en el tránsito de la política a la biopolítica, cuando la primera asumió cabalmente la tarea de disciplinar los cuerpos (a lo que Foucault llama anatomopolítica) como un mecanismo para administrar a las poblaciones (a lo que Foucault llama biopolítica). ${ }^{32}$ Como afirma Foucault, durante mucho tiempo y hasta ahora, la institución de la familia ha brindado un apoyo decisivo para la aplicación efectiva de políticas orientadas a la población para controlar (promover o desalentar) la natalidad. ${ }^{33}$

31. Michel Foucault, The Will to Knowledge. History of Sexuality, vol. I (Nueva York: Pantheon Books, 1978), 84. Versión consultada en inglés, la traducción es mía.

32. Ibíd., 100 .

33. Ibíd., 58. 
Como contrapunto a las ideas de Foucault sobre biopolítica, Silvia Federici, al proporcionar una interpretación feminista y marxista de la infame e histórica caza de brujas, explica cómo en la Europa de los siglos XVI al XVIII, las matronas, vecinos y familiares tenían la obligación de vigilar a las mujeres, reportando sobre cualquier nuevo nacimiento, e incluso de examinar a las mujeres "sospechosas de haber dado a luz en secreto". ${ }^{34} \mathrm{El}$ análisis de Federici supone, como ella misma afirma, "una crítica a la teoría del cuerpo de Michel Foucault [quien] ignora el proceso de reproducción, [y] funde las historias femenina y masculina en un todo indiferenciado". ${ }^{35}$ Aunque Federici no llega a expresarlo de ese modo, su análisis pone en jaque la distinción que Foucault hace entre la anatomopolítica que disciplina y optimiza las capacidades corporales y la biopolítica que regula y administra a la población, ${ }^{36}$ porque desde la perspectiva de la reproducción, cuando el cuerpo es femenino, la optimización de las capacidades corporales y la regulación de la población van por el mismo camino.

En cierto sentido, Marcia, que representa este ejercicio del poder sobre otros cuerpos, también problematiza las políticas de la familia. Ella controla y amenaza a su hermana para conseguir una niña perfecta, una de la cual disfruta, mientras que está siendo cuidada por alguien más.

\section{Alicia. El homo saCer es ella: \\ EL CUERPO FEMENINO COMO NUDA VIDA ${ }^{37}$}

Antes de entrar al personaje ya anunciado de la niña, Karlita, que cierra la reflexión en torno a lo que podría ser una trayectoria de la biopolítica, hacemos una parada por el "Cuarto de las chicas", lugar en el que, según narra el guion, se realizan los procedimientos. Allí encontramos a Alicia, una niña de 14 años (algo mayor en las primeras versiones del guion) que acude sola a esa hacienda perdida en medio de los Andes para que Isabel le practique un aborto. El cuerpo joven de la niña no reacciona ante el procedimiento como se hubiera esperado, y la historia de "A puerta cerrada" la encuentra agonizando en el cuarto de las chicas. ${ }^{38}$

Considerando el marco legal y constitucional que rige en Ecuador respecto de los derechos reproductivos, la pregunta que articula esta reflexión sería: ¿puede el

34. Silvia Federici, Caliban and the Witch. Women, The Body and Primitive Accumulation (Nueva York: Autonomedia, 2004), 84.

35. Ibíd., 17.

36. Foucault, The Will to Knowledge, 58.

37. Se usará Vida Desnuda o Nuda Vida en este texto.

38. Que coincide con el título en inglés del guion, "The Ladies Room". 
giro hacia la tanatopolitica propuesto por Giorgio Agamben ${ }^{39}$ ser útil para analizar el estatus político de aquellas niñas y mujeres que, en Ecuador, se practican abortos consentidos, clandestinos, muchas veces en condiciones de riesgo vital?

Alicia, la niña de 14 años que se desangra sola dentro del cuarto de las chicas propone una paradoja: si bien en virtud de los artículos constitucionales 44 y 45, citados en la primera sección de este análisis, sus derechos prevalecerían por sobre aquellos de los otros sujetos, su condición de gravidez determina que conforme a la interpretación tradicional del texto constitucional, otro sujeto de derechos ${ }^{40}$ también favorecido por el principio de interés superior, habita su cuerpo. Dado que la tipificación del aborto no punible en el art. 150 del Código Orgánico Integral Penal del Ecuador no contempla entre sus causales la prematura edad de la gestante, el interés superior de Alicia estaría matizado por la penalización del acto. Dicho de otro modo, en el contexto jurídico-político del Ecuador, el interés superior de la niña deja de ser considerado superior y sus derechos fundamentales cesan de ser considerados tales en el momento en el que se confirma su embarazo. Así, Alicia está muriendo, encerrada dentro del cuarto en el que se le practicó el procedimiento; mientras Isabel y Marcia hacen todo lo posible por salvarle la vida; todo, excepto buscar atención médica por miedo a ser denunciadas y apresadas.

Pero, si el interés superior de Alicia desaparece en la coyuntura, provocando el virtual desvanecimiento de ciertos derechos que le asisten por su condición de niña ante la ley, algo similar, aunque la Constitución ecuatoriana garantiza a todas las personas el derecho a tomar decisiones libremente sobre su salud reproductiva, como hemos discutido, este derecho está efectivamente limitado para las mujeres por el postulado constitucional de inviolabilidad de la vida desde la concepción. De este modo, la Constitución establece un mecanismo de exclusión que, en lo relativo a los derechos reproductivos, configura a las mujeres ${ }^{41}$ como una excepción respecto del ejercicio de esos derechos reconocidos a todas las personas.

Entonces, podría decirse que, con relación a los derechos reproductivos, la norma constitucional incluye a las mujeres a través de una forma de ser excluidas, estableciendo lo que Agamben denomina una relación de excepción. ${ }^{42}$ Reflexionando sobre

39. Giorgio Agamben, Homo sacer: sovereign power and bare life (Stanford: Stanford University Press, 1998).

40. Esta forma de enunciación no implica una toma de posición de la autora respecto del reconocimiento de la personalidad del producto de la concepción, durante el proceso de embarazo; sino más bien, la comprensión de que el marco constitucional del Ecuador y el marco regional de derechos humanos consideran jurídicamente a tal un sujeto al cual es posible arrogarle derechos.

41. Ciertas mujeres, las que experimentan un embarazo no deseado.

42. Agamben, Homo sacer, 18. 
esta figura, Agamben explica que "llamamos relación de excepción a esta forma extrema de la relación que solo incluye algo a través de su exclusión". ${ }^{43}$

Lo que se incluye a través del mecanismo de exclusión es precisamente la vida desnuda, la que carece de derechos, la que queda a merced de la ley, porque no puede evitarlo. ${ }^{44}$ Esta vida desnuda o nuda vida se representa en el guion a través del cuerpo de una niña, o (para que leerlo no resulte tan doloroso), de una joven mujer que, sometida irremediablemente a la violencia y un poder que la perenniza, se desangra en una habitación lejos de los discursos acerca del derecho de niñas y mujeres a una vida digna, a salud integral, a decidir cuándo y cuántos hijos e hijas tener y, por consiguiente, a reclamar su pertenencia irrestricta dentro de la categoría de todas las personas a quienes el texto constitucional reconoce estos derechos. Alicia se desangra a puerta cerrada, porque, como dijera Walter Benjamin, "la sangre es el símbolo de la vida [desnuda]". ${ }^{45}$

\section{KARLITA, SER-DESPUÉS DE-NACER: ${ }^{46}$ LA VIDA COMO POSIBILIDAD}

Karlita, la niña que nació 6 años atrás producto de un embarazo no deseado forzado a término, es el personaje más joven de "A puerta cerrada", esta historia que articula una reflexión respecto de temas relacionados con derechos de las mujeres y justicia reproductiva desde una perspectiva biopolítica. Karlita constituye la última parada (previsible a la fecha de escritura de este texto) en lo que podría ser considerada una trayectoria del pensamiento biopolítico: Karlita es el sujeto geneapolitico. ${ }^{47}$

Hasta aquí la reflexión se ha concentrado en las circunstancias que rodean dos diferentes formas de enfrentar un embarazo no deseado ${ }^{48}$ en Ecuador: maternidad coerciti-

43. Ibíd., 31 .

44. Ibíd., 28-9.

45. Walter Benjamin, "Critique of Violence", en Reflections: essays, aphorisms, autobiographical writings, ed. Peter Demetz, trad. Edmund Jephcott (Nueva York: Schocken Books, 1986), 297.

46. Este término es una propuesta de Chiara Bottici que se desarrolla a lo largo de esta sección del texto; sin embargo se deja constancia de que la traducción literal (a nivel lingüístico) del término, desde el idioma inglés en que fuera originalmente acuñado, sería seres-después del-nacimiento; se acoge la fórmula de redacción seres-después de-nacer por considerarse equivalente a nivel semántico y más apropiada a nivel pragmático; el nacimiento es un evento, el nacer implicación acción y, por tanto, agencia.

47. Neologismo compuesto por dos raíces griegas que significan nacimiento y política, desarrollado por Chiara Bottici en su texto "Rethinking the Biopolitical Turn: From the Thanatopolitical to the Geneapolitical Paradigm", Graduate Faculty Philosophy Journal, vol. 36, n. ${ }^{\circ} 1$ (2015).

48. Vale la pena aclarar que en el marco de este texto, y de la investigación a la que se refiere, se considera embarazo no deseado (unwanted pregnancy, en inglés) a aquel en el cual una mujer en estado de gravidez 
va o aborto clandestino. A partir de aquí pregunto: ¿qué es una hija no deseada? ¿Qué implica para Karlita el ser una hija no deseada? ¿Qué y quién es Karlita?

Para ensayar una respuesta, es necesario aclarar que un hijo o, en lo relativo a nuestra historia, una hija no deseada, sería la hija que ha nacido de un embarazo no deseado coercitivamente llevado a término. Una hija producto de un embarazo con tales características, sería no deseada a partir del nacimiento, solo si la madre biológica siente con respecto de la recién nacida, un rechazo similar al que sintió con respecto de su embarazo (y por las mismas razones). Siendo así, una hija no deseada será no deseada primaria y especialmente con relación a su madre biológica. Y esa niña muy probablemente experimentará esa especie de rechazo que marca sus circunstancias, mientras la madre biológica sea su cuidadora principal.

Pero más allá de esa decisiva, íntima e intersubjetiva esfera, reproducida una y otra vez en un Ecuador en el que, de acuerdo a la Tasa de Fecundidad No Deseada, Karlita es 1 de cada 6 niños y niñas en el país, ${ }^{49}$ esa hija no deseada es nada más y nada menos que una niña. Una niña. Una vida que ocurre cuando un cuerpo se separa de otro cuerpo después del nacimiento, pura inmanencia, como anota Gilles Deleuze, "UNA VIDA, y nada más". ${ }^{50}$

No es una coincidencia que Deleuze propusiera dos ejemplos para explicar sus ideas acerca de una vida como inmanencia pura: 1. la vida desnuda en la forma del homo tantum (solo un hombre), que está muriendo; 2 . los niños pequeños, que a decir de Deleuze están "imbuidos por una vida inmanente que es pura potencia e incluso beatitud". ${ }^{51}$ El primer ejemplo se refiere directamente al personaje de Alicia; el segundo, a Karlita. "Una vida solo contiene virtuales" escribe Deleuze, explicando que lo virtual no es algo que carezca de realidad, sino el evento o singularidad en el proceso

no desea ni al embarazo ni el producto de esa gestación. Si en algún punto en el curso de la gestación la mujer cambia de opinión y elige voluntariamente ese embarazo, este no puede ser considerado no deseado y pasará a ser visto como embarazo no planificado o embarazo a destiempo, que corresponden respectivamente a las categorías de unintended pregnancy y mistimed pregnancy en inglés. Las particularidades de estos $-\mathrm{y}$ sus diferencias respecto de los no deseados (todas con implicaciones distintas para la mujer gestante) están bastante claras en los estudios de población y de salud pública en otras partes del mundo (ver Singh, Sedgh y Hussain, "Unintended pregnancy: worldwide levels, trends, and outcomes", Studies in family planning, vol. 41, n. 4 (2010): 241-50. En Ecuador, hasta el momento, no han sido establecidas claramente tales distinciones ni aun en instrumentos oficiales en materia de salud pública.

49. Ministerio de Salud Pública del Ecuador e INEC, Encuesta Nacional de Salud y Nutrición, tomo II, Salud Sexual y Reproductiva, 119.

50. Gilles Deleuze, "Immanence: A Life", en Pure Immanence. Essays on A Life (Nueva York: Zone books, 2012), 27. Versión consultada en inglés.

51. Ibíd., 28-31. 
de actualización. También afirma que "al evento considerado como no actualizado (indefinido) no le falta nada". ${ }^{2}$

Las virtualidades que pueblan una vida, pura inmanencia son la multiplicidad de potencialidades para (ser, estar o hacer) y potencialidades para no (ser, estar o hacer); potencialidades que Karlita, como cada niño y niña, representa. Consecuentemente, Karlita es la vida en potencia, enmarcada en ese rechazo que experimenta al tener que vivir con una mujer (Isabel) para quien la niña es el testimonio de su propia impotencia. Un día Karlita se convertirá en una mujer adulta que, paradójicamente, luego de haber sido la vida en potencia, es posible que llegue a entender el significado de la vida (de una mujer) como impotencia.

En sintonía con estas ideas, desde la filosofía política, Chiara Bottici reflexiona acerca de ese giro biopolítico que marca un trayecto desde la biopolítica de Foucault hacia la tanatopolítica de Agamben. Bottici considera que esta trayectoria de comprensión de la biopolítica, de política sobre la vida a política sobre la muerte, obedece a una cierta tendencia de la filosofía occidental: concebir a los seres humanos como seres-hacia-la muerte criaturas que ven en el hecho de morir el único horizonte ontológico posible.

A decir de Bottici, informada por reflexiones de Adriana Cavarero, ${ }^{53}$ esta conciencia de la muerte como el final del camino nos convierte en criaturas monádicas, solitarias, porque "el cuerpo muere solo" ${ }^{54}$ Frente a esta filosofía (y política) del ser que encuentra en la muerte el horizonte ontológico de una vida en soledad, Bottici, construyendo sobre las premisas de Cavarero, propone una alternativa: repensar a los humanos como seres-después de-nacer, desplazando así el punto de inflexión de nuestra naturaleza ontológica desde la muerte (un evento que el cuerpo experimenta inevitablemente solo) hacia el nacimiento (un evento que el cuerpo experimenta inevitablemente acompañado).

Este desplazamiento procura poner en evidencia que "llegamos a la escena literalmente junto con un-otro ser humano". ${ }^{55}$ Esta alteración del punto de vista, Bottici argumenta, podría recentrar nuestra idea del ser, reivindicando en nuestra dimensión ontológica el valor del acompañamiento; para así definirnos irremediablemente como seres relacionales. Este gesto permitiría también reclamar la prioridad del nacimiento,

52. Ibíd., 31.

53. Adriana Cavarero, “ “A Child Has Been Born unto Us': Arendt on Birth”, Philosophia. A Journal of Continental Feminism, vol. 4, nº 1 (2014).

54. Chiara Bottici, Imaginal Politics: Images beyond Imagination and the Imaginary, New Directions in Critical Theory (Nueva York: Columbia University Press, 2014), 68.

55. Ibíd. 
entendido como nuestra experiencia primordial en tanto que seres por sobre la muerte; al respecto Bottici concluye que "podemos ser seres-hacia-la muerte solo porque somos seres-después de-nacer". ${ }^{56}$

Estas reflexiones acerca de los seres-después de-nacer darían lugar a una nueva dirección en esa trayectoria biopolítica, para lo cual Bottici acuña el neologismo geneapolítico, del griego genea que significa nacimiento.

Para Bottici, lo geneapolítico reconoce esa "prioridad ontológica y política del nacimiento sobre la muerte" ${ }^{57} \mathrm{y}$ abre la posibilidad de articular una nueva forma de comprensión de la biopolítica, alineada con lo que Roberto Espósito concibiera como una biopolítica afirmativa, que ya no es entendida como una forma de poder sobre la vida, sino como una forma de poder de la vida ${ }^{58} \mathrm{y}$, si acaso, una forma de poder desde la vida. Aquí, el horizonte existencial de los seres humanos, en tanto que seres-después de-nacer ya no sería la muerte, sino precisamente el nacer, como acto relacional, que constituye, además, el evento inaugural de un camino enteramente por definir. En palabras de Cavarero, nacer sería "el inicio de un viaje todavía no identificado, un evento de pura posibilidad" 59 que da cuenta de esa relacionalidad constitutiva que marca la vulnerabilidad propia de actores y actrices involucradas en el "escenario de la natalidad". ${ }^{60}$ Así, todo ser-después de-nacer, vulnerable y en potencia a la vez, requiere un cuidado también relacional.

En el contexto de la historia de A puerta cerrada Karlita sería, en términos de Bottici, un ser-después de-nacer, el sujeto geneapolítico que representa el poder de la vida; para Deleuze, ella es precisamente eso, UNA VIDA, "pura potencia e incluso beatitud"; y para Cavarero pura posibilidad frente a quien, una vez nacida, existe una innegable obligación de protección, crianza y cuidado, sin que en el contexto legal ecuatoriano haya claridad respecto de quien, además de la madre biológica, ${ }^{61}$ es el conminado a cumplir con esta obligación.

\section{A MODO DE CONCLUSIÓN}

En este texto se ha propuesto a la biopolítica como una manera de entender los modos de ejercicio del poder sobre la vida, lo cual nos ha permitido, hasta cierto

56. Ibíd.

57. Bottici, "Rethinking the Biopolitical Turn".

58. Roberto Esposito, Bios: Biopolitics and Philosophy (Minnesota: University of Minnesota Press, 2008), 56.

59. Cavarero, " 'A Child Has Been Born unto Us': Arendt on Birth”, 15.

60. Ibíd., 28.

61. Y acaso el padre, pero esa reflexión nos llevaría en otras direcciones. 
punto, rastrear estas formas de concebir la relación entre el poder y lo vital. Así, a través de Isabel (el sujeto biopolítico) y Marcia (el poder biopolítico), el diseño de personajes del guion cinematográfico A puerta cerrada asumió como punto de partida la biopolítica de Foucault, matizada por las reflexiones de Federici y las nociones de maternidad coercitiva y vigilancia infinitesimal.

A partir de allí se procuró hacer una segunda parada en esa trayectoria biopolítica, para acercarnos, de la mano de Alicia (el sujeto tanatopolítico), a la tanatopolítica de Agamben y a la realidad de esas niñas mujeres que, en Ecuador, se someten a abortos clandestinos. Y finalmente, se acabó de configurar esa trayectoria biopolítica con el personaje de Karlita (el sujeto geneapolítico), quien también nos permite marcar una traslación hacia esa biopolítica afirmativa propuesta por Espósito, que ya no se refiere al poder sobre la vida, sino al poder de la vida.

Varias cuestiones quedan por resolverse. Por un lado, la relativa exclusión de las mujeres de esa categoría de todas las personas, que garantiza el reconocimiento de derechos constitucionales; como hemos visto, algunos derechos constitucionalmente reconocidos dejan de ser considerados fundamentales para las mujeres una vez confirmado el embarazo. Por otro lado, debe revisarse (corregirse) la situación de abandono ante la autoridad y la ley, de aquellas niñas y mujeres que, en Ecuador, se someten a abortos clandestinos; su situación es especialmente preocupante si en el horizonte de entendimiento propuesto en el caso del personaje de Alicia, asumimos que frente a estas niñas y mujeres la ley opera en un particular modo o estado de excepción. Y finalmente, es necesaria una reflexión profunda en torno a quién debe cumplir la obligación de proteger, criar y cuidar a ese ser-después de-nacer, cuya existencia es impuesta a través de la coerción que fuerza a término un embarazo no deseado. ¿Será que realmente tal obligación corresponde a la persona a quien esa vida le fue impuesta y es, por tanto, testimonio de su propia impotencia?, ¿o a la persona o personas que representan las fuerzas y poderes que forzaron ese embarazo y nacimiento? y, de ser el segundo caso, ¿de qué modo se lograría efectivamente el cumplimiento de tales operaciones?

Frente a estos seres-después de-nacer, nacidos en circunstancias de violencia muy particulares, es un imperativo histórico resolver cómo cumplir esta obligación de cuidado en un escenario que no siga vulnerando los derechos de esas mujeres y, peor aún, niñas, que han sido forzadas a ser madres por el actual marco jurídico (y otras formas de poder sociopolítico) en Ecuador y otros puntos de Latinoamérica.

Además, resulta indispensable empezar a tomar distancia de esas políticas sobre la vida, que, como diría Foucault, administran lo viviente, imponiendo la natalidad (según Federici) o la muerte (según Agamben), para empezar a preguntarnos de qué modo, con qué herramientas, desde qué voluntades y con qué objetivos se puede articular esas políticas de y desde la vida. 
Es importante anotar que algunas de estas cuestiones encuentran en el guion cinematográfico A puerta cerrada una respuesta-propuesta absolutamente ficticia e imaginaria; sin embargo, por la naturaleza misma de la obra artística que se ha elegido para articular este proyecto de investigación basado en la práctica artística, para conocer tal respuesta-propuesta será necesario esperar a que algún día ese guion logre construirse un camino hacia las pantallas.

\section{BIBLIOGRAFÍA}

Agamben, Giorgio. Homo sacer: sovereign power and bare life. Traducido por Daniel HellerRoazen. Stanford: Stanford University Press, 1998.

-. Homo sacer. El poder soberano y la nuda vida. Valencia: Pre-Textos, 1998.

Bakunin, Mijaíl. La libertà degli uguali. Milan: Elèuthera, 2009.

Benjamin, Walter. "Critique of Violence". En Reflections: essays, aphorisms, autobiographical writings, editado por Peter Demetz, traducido por Edmund Jephcott, 277-300. Nueva York: Schocken Books, 1986.

Bottici, Chiara. Imaginal Politics: Images beyond Imagination and the Imaginary. Nueva York: Columbia University Press, 2014.

- "Rethinking the Biopolitical Turn: From the Thanatopolitical to the Geneapolitical Paradigm". Graduate Faculty Philosophy Journal. Vol. 36, n. ${ }^{\circ} 1$ (2015): 175-97.

Cavarero, Adriana. " 'A Child Has Been Born unto Us': Arendt on Birth". Philosophia. A Journal of Continental Feminism. Vol. 4, n. ${ }^{\circ} 1$ (2014): 12-30.

Deleuze, Gilles. "Immanence: A Life". En Pure Immanence. Essays on A Life, editado por Gilles Deleuze, traducido por Anne Boyman, 25-33. Nueva York: Zone Books, 2012.

Ecuador. Constitución de la República del Ecuador. Registro Oficial 449, 20 de octubre de 2008.

—. Código Orgánico Integral Penal. Registro Oficial Suplemento 180, 10 de febrero de 2014.

Esposito, Roberto. Bios: Biopolitics and Philosophy. Minnesota: University of Minnesota Press, 2008.

Federici, Silvia. Caliban and the Witch. Women, The Body and Primitive Accumulation. Nueva York: Autonomedia, 2004.

Foucault, Michel. The Will to Knowledge. History of Sexuality. Vol. I. Nueva York: Pantheon Books, 1978.

Galarza, María Teresa. "Dissenting fiction re-righting law: practice-led research into biopolitics, women's rights and reproductive justice in Ecuador". Tesis doctoral, The University of Melbourne, 2017. 〈https://minerva-access.unimelb.edu.au/handle/11343/210743).

Leavy, Patricia. Fiction as Research Practice: Short Stories, Novellas, and Novels. Walnut Creek, CA: Left Coast Press Inc., 2013. 
Ministerio de Salud Pública del Ecuador. Indicadores Básicos de Salud 2010. Quito: MSP / INEC / Organización Panamericana de la Salud, 2011.

—. Indicadores Básicos de Salud 2012. Quito: Ministerio de Salud Pública, 2013.

- Plan Nacional de Salud Sexual y Salud Reproductiva. Quito: Viceministerio de Gobernanza de la Salud Pública, 2017.

Ministerio de Salud Pública del Ecuador e INEC. Encuesta Nacional de Salud y Nutrición. Tomo II. Salud Sexual y Reproductiva. Quito: Ministerio de Salud Pública / INEC, 2015.

O'Reilly, Andrea. Matricentric Feminism. Theory, Activism, and Practice. Ontario: Demeter Press, 2016.

Paul, Alvaro. "Controversial Conceptions: The Unborn and the American Convention on Human Rights". Loyola University Chicago International Law Review. Vol. 9, Issue 2 (2012): 209-47.

Rancière, Jacques. Dissensus: On Politics and Aesthetics. London: Continuum, 2010.

Ross, Loretta J., y Rickie Solinger. Reproductive justice. An introduction. Oakland: University of California Press, 2017.

Ruddick, Sara. Maternal Thinking. Toward of Politics of Peace. Boston: Beacon Press, 1995.

Zaragocin, Sofía, María Rosa Cevallos, Guglielmina Falanga, Iñigo Arrazola, Gabriela Ruales, Verónica Vera y Amanda Yépez. "Mapeando la criminalización del aborto en el Ecuador". Revista de Bioética y Derecho, n. 43 (2018): 109-25. 〈http://scielo.isciii.es/pdf/bioetica/ n43/1886-5887-bioetica-43-00109.pdf . 